\section{MedienPädagogik}

www. medienpaed.com
Zeitschrift für

Theorie und Praxis

der Medienbildung

ISSN 1424-3636

Themenheft Nr. 18: Neue Medien und individuelle Leistungsdarstellung Möglichkeiten und Grenzen von ePortfolios und eAssessments

\title{
Mit ePortfolios selbstgesteuert lernen Ein Ansatz zur Reflexionsförderung im Rahmen eines hochschulweiten ePortfoliosystems
}

Thomas Czerwionka, Sönke Knutzen und Detlev Bieler

\begin{abstract}
Der vorliegende Artikel beschreibt einen Ansatz zur medienbasierten Reflexionsunterstützung Studierender. Ausgehend vom lebenslangen Lernen und dessen individuellen Anforderungen wird zunächst näher auf selbstgesteuertes Lernen und den Stellenwert von Reflexionsfähigkeit eingegangen. Ein Modell lernbezogener Einflussvariablen dient anschliessend der Verdeutlichung verschiedener möglicher Reflexionsebenen, -gegenstände und -ziele. Am Beispiel von (e)Portfolios werden daraufhin Möglichkeiten mediengestützter Reflexionsförderung beschrieben, bevor schliesslich die reflexionsunterstützenden Massnahmen im ePortfoliosystem der Technischen Universität Hamburg-Harburg dargestellt werden.
\end{abstract}

\section{$1 \quad$ Einleitung}

Gesellschaftliche, wirtschaftliche, wissenschaftliche und technische Wandlungsprozesse machen die Ermöglichung lebenslangen Lernens zu einem wesentlichen Bildungsziel, an das vielfältige strukturelle und individuelle Anforderungen geknüpft sind. Lebenslanges Lernen, verstanden als formales, nicht-formales und informelles Lernen über alle Lebensphasen hinweg, erfordert nicht nur eine entsprechende Infrastruktur, sondern auf Seiten der Lernenden insbesondere die Fähigkeit, sich der eigenen Lernbedürfnisse und -voraussetzungen bewusst zu werden und selbst über angemessene Ziele, Formen und Wege des Lernens zu entscheiden.

Im bildungspolitischen Kontext werden ein von den Lernenden weitgehend selbst verantwortetes Lernen und die dazu notwendigen Kompetenzen z. B. als Entwicklungsschwerpunkte in der BLK-Strategie für lebenslanges Lernen aufgeführt: Während Kindheit und Jugendalter noch von fremdorganisierten Lernangeboten und der Pflicht zum Lernen geprägt sind, steigt mit zunehmendem Alter der Anteil des eigenverantwortlich zu gestaltenden Lernens - so auch für junge Erwachsene, die sich für ein Hochschulstudium entschieden haben (vgl. BLK 2004, S. 13 ff.). Die bildungspolitischen Ziele finden sich in aktuellen hochschuldidaktischen Grundsätzen wieder. Hier markieren die Ausrichtung des Studiums an Kompetenzen (Outcome- statt Input-Orientierung) und eine lernerzentrierte Lehrauffassung zwei im Hinblick auf die Befähigung zu selbstgesteuertem lebenslangen Lernen rele- 
vante Komponenten. Lernförderung und aktives Lernen sollen dabei im Mittelpunkt stehen.

\section{Selbstgesteuertes Lernen}

Der Ansatz des selbstgesteuerten Lernens gewinnt in der allgemeinen und beruflichen Bildung wie in der Hochschullehre an Bedeutung. Mit ihm ist die Ansicht verbunden, dass Lernen nicht mit einem formalen Bildungsabschluss endet, sondern darüber hinaus - in der Regel berufsbegleitend - fortgesetzt wird. In der Konsequenz bedeutet dies, dass Lernprozesse zunehmend von den Lernenden selbst gesteuert werden (vgl. Dohmen 1999, S. 20), was insbesondere die Hochschullehre, die häufig Vorlesungscharakter hat, vor Herausforderungen stellt. Dohmen schreibt, dass es keine Alternative "zu einem offeneren, mehr von den Lernenden selbst gesteuerten Lernen» (ebd., S. 18) gibt, wenn es die Bildungspolitik ernst meint mit der «Verwirklichung des notwendigen bewussteren, lebenslangen Lernens Aller'» (ebd.).

Zu der verstärkten Selbsttätigkeit der Lernenden kommt zusätzlich eine Erweiterung der Lernorte und Lernzeiten. Die Bildungsinstitutionen sind nicht mehr hauptverantwortlich für die Organisation der Lernprozesse und die Wissensvermittlung. Lernen findet vielmehr überall statt, es wird entgrenzt. Wissen muss «überall erworben bzw. erarbeitet werden» (ebd., S. 21).

Im Folgenden wird der Begriff der Selbststeuerung in aller Kürze erläutert und im Kontext erziehungswissenschaftlicher Konzepte dargestellt.

\section{Selbststeuerung und selbstgesteuertes Lernen}

Häcker (2007a) bezeichnet Selbststeuerung als «eines der prominentesten Zielkriterien, die gegenwärtig im Zusammenhang mit der Debatte um die Verbesserung der Qualität unterrichtlichen Lernens genannt werden» (S. 62), wenngleich der Begriff in der erziehungswissenschaftlichen Diskussion unscharf ist. In der Literatur werden unterschiedliche Begriffe mit Selbststeuerung synonymisiert: Selbstorganisation, Selbsttätigkeit, Autonomie, autodidaktisches Lernen, Selbstbestimmung, Selbstregulation usw. (vgl. ebd., S. 63; Faulstich/Zeuner 1999, S. 141). Um einen kurzen Überblick über die zum Teil gegensätzlichen Auffassungen zu geben, sind im Folgenden einige Positionen zusammengefasst:

- Siebert (2006) verwendet den Begriff der Selbstorganisation als «systemtheoretische[n] Oberbegriff, während Selbststeuerung als pädagogischer Begriff vor allem für Lernprozesse verwendet wird» (S. 107).

- Friedrich/Mandl (1997) begrenzen die Selbststeuerung auf die Frage nach dem Wie und Wann der Lernregulation, wogegen sich die Selbstbestimmung der Lernenden auf die Auswahl der Inhalte beziehen kann (vgl. S. 239). 
- Bastian (2003) vertritt die Gegenposition, indem er Selbststeuerung über Selbstbestimmung einordnet (vgl. S. 3).

- Dohmen (1999) bezeichnet selbstgesteuertes Lernen als «eine besondere Form des für Fremdunterstützung offenen, Selbstlernens'» (S. 16). Dazu gehört nicht nur die Selbstverarbeitung der Informationen und Eindrücke, sondern auch, dass das Lernen nicht primär von anderen gesteuert wird. Damit konzentriert es sich auf das «emanzipatorische» Bildungsziel, dass erwachsene Lerner lernen sollen, ihre Lernprozesse so weit wie möglich selbst zu steuern (vgl. ebd.).

- Arnold/Gómez Tutor (2007) definieren selbstgesteuertes Lernen als «einen aktiven Aneignungsprozess mit umfassenden Möglichkeiten zur eigenen Ausrichtung (Bedarfsdefinition, Zielsetzung etc.), zur Unterstützungsnutzung (Lernressourcen etc.) sowie zur Überprüfung im Lernprozess» (S. 54).

\section{Selbstgesteuertes Lernen in erziehungswissenschaftlichen Konzepten}

Selbstgesteuertes Lernen hat in vielen Epochen als Idee bzw. als methodischer Zugang eine Rolle gespielt (vgl. Arnold/Gómez Tutor 2007, S. 123). Dies geht zurück zur Reformpädagogik und darüber hinaus bis zum Humanismus im 16. Jahrhundert.

Die Konzepte der Reformpädagogik sowie der Kognitionstheorie von Piaget u. a. werden «bestätigt, ergänzt und radikalisiert» (Siebert 2006, S. 21) durch den Konstruktivismus. Entgegen den Instruktionsmethoden der traditionellen Didaktik bevorzugt der (gemässigte) Konstruktivismus Methoden, welche «die Wirklichkeitskonstruktion der Lernenden thematisieren, reflektieren und erweitern» (ebd., S. 88). Wesentliches Merkmal dieser Didaktik ist neben Situiertheit, Anschlussfähigkeit und Biografieorientierung die Selbststeuerung. Lernen wird als ein aktiver Aneignungsprozess verstanden, wobei das Individuum über sein Lernen, seine Lernbedürfnisse, aber auch über Hinzuziehung der notwendigen Ressourcen inklusive professionellen Lernangeboten oder Lernhilfen entscheidet. Die traditionelle «Belehrungsdidaktik» verliert zugunsten einer «Ermöglichungsdidaktik» in oben genanntem Sinne zunehmend an Bedeutung. Die Ermöglichungsdidaktik ist die didaktische Theorie im Sinne des selbstgesteuerten Lernens, wobei die Lehre das prinzipiell selbstgesteuerte Lernen lediglich ermöglichen kann. Lehrende übernehmen statt der Rolle der Wissensvermittler die Rolle von Beratern oder Coachs. Die Lernumgebungen und Lernsituationen, die den Lernenden bereitgestellt werden, müssen dabei anregend gestaltet und arrangiert werden (vgl. Siebert 2006, S. 86 ff.; Arnold/Gómez Tutor 2007, S. 50/S. 125; Arnold 1993, S. 53).

\section{«Selbstlernkompetenzen»}

Um selbstgesteuert lernen zu können, müssen die Lernenden bestimmte Fähigkeiten und Voraussetzungen mitbringen bzw. sich erarbeiten. Arnold/Gómez Tutor (2007) nennen als wesentliche Handlungskompetenzen die Sach-, Selbst- und So- 
zialkompetenz. Dazu kommen im Zusammenhang mit Fachkompetenz die Methodenkompetenz, als Selbstkompetenz die emotionale bzw. personale Kompetenz sowie die kommunikative Kompetenz (vgl. S. 126).

Besonderen Stellenwert hat die methodische Kompetenz hinsichtlich der Reflexion des Lernprozesses (vgl. ebd., S. 127). Die lernende Person muss sich «über die eigenen Aktivitäten als verursachende Instanz des Lernprozesses gewiss sein [und erhält] dadurch auch eine positive Rückkopplung über ihre eigene Lernfähigkeit» (ebd.). Dazu gehören als Eigenschaften kommunikativer Kompetenz die sprachliche Ausdrucksfähigkeit sowie die Dialogfähigkeit, «denn ohne den Austausch mit bzw. die Unterstützung von anderen und damit ihre Impulse und Perturbierungen schreitet der Lernprozess nicht voran» (ebd.). Wichtig für erfolgreich selbstgesteuerte Lernprozesse ist auch die emotionale Kompetenz. Diese beinhaltet die Fähigkeit, die eigenen Gefühle zu verstehen und zum Ausdruck bringen zu können als eine Voraussetzung für die Reflexion über das eigene Lernen.

\section{Lernen und Reflexion}

Die beschriebenen individuellen Anforderungen selbstgesteuerten Lernens verdeutlichen die zentrale Bedeutung der Reflexionsfähigkeit. Soll diese Fähigkeit zielgerichtet gefördert werden, muss zunächst der Begriff der Reflexion näher beschrieben und hinsichtlich des Lernens konkretisiert werden. Für die praktische Umsetzung reflexionsunterstützender Elemente im Lernen ist darüber hinaus ein Blick auf entsprechende empirische Ergebnisse sinnvoll.

\section{Dimensionen des Reflexionsbegriffs}

Im Allgemeinen wird unter Reflexion das Nachdenken einer Person über etwas verstanden. In Bezug auf die erste der hiermit angesprochenen Dimensionen, die der beteiligten Individuen, kann unterschieden werden zwischen a) der Selbstreflexion, an der neben der reflektierenden Person keine weitere/n beteiligt ist/sind, und b) der Reflexion im Austausch mit anderen. Die zweite Dimension betrifft den Reflexionsgegenstand. Der Begriff der Selbstreflexion wird üblicherweise gewählt, wenn die reflektierende Person Aspekte ihres Selbst thematisiert. Setzen sich weitere Personen ebenfalls mit Aspekten einer einzelnen (selbstreflektierenden) Person auseinander, kann von Fremdreflexion gesprochen werden. Demgegenüber sind in sog. Gruppen- oder Teamreflexionen grundsätzlich eher gruppenbezogene Aspekte wie z. B. gemeinsame Zielvorstellungen oder das sozial-emotionale Klima Gegenstand der Reflexion (vgl. Siebert 1991, S. 22 f.; Wyss 2008, S. 3 ff.). Die Wahl des Reflexionsgegenstands hängt ab von einer dritten Dimension, dem Reflexionsziel. Der Zielbezug verdeutlicht, dass Reflexion immer sowohl rückblickenden als auch vorausschauenden Charakter hat. 


\section{Lernbezogene Reflexion}

Im Hinblick auf Lernen findet sich in der Literatur eine Vielzahl möglicher Reflexionsziele und -gegenstände. So soll lernbezogene Reflexion z. B. dazu dienen, sich des Sinns und Zwecks des Lernens bewusst zu werden, Eigenverantwortungs- und Selbststeuerungsanteile im Lernen zu erhöhen oder schlicht das eigene Lernen effektiver oder effizienter zu gestalten. Das jeweils angestrebte Ziel lässt sich durch die Wahl des Reflexionsgegenstands in unterschiedlichster Weise spezifizieren; Beispiele mit Lernbezug sind persönliche Lernziele, -motive und -bedarfe, das eigene Lernvermögen und -verhalten, Lernerfahrungen, -fortschritte und -hemmnisse, Lernprozesse, -methoden und -strategien, Lernwirkungen und -produkte sowie Lerninhalte bzw. -gegenstände.

Die mannigfaltigen Bezüge von Reflexion im Lernen lassen sich veranschaulichen, indem Lernen im Sinne des Modells der vollständigen Handlung als gesteuerter Prozess verstanden wird, der die drei Teilprozesse Planung, Durchführung und Bewertung umfasst. Diese Teilprozesse bzw. Phasen unterliegen sowohl selbst- als auch fremdgesteuerten Einflüssen und können im Lernprozess mehrfach und in unterschiedlichen Kombinationen durchlaufen werden. In allen Phasen des Lernprozesses spielen sich einander beeinflussende kognitive, metakognitive, emotionale, motivationale und volitionale Aspekte eine Rolle. Im Einzelnen können den drei Phasen auf Seiten der Selbststeuerungseinflüsse die folgenden Aktivitäten zugeordnet werden (vgl. Schiefele/Pekrun 1996, S. 269 ff.; Wild/Hofer/Pekrun 2001, S. 211 ff.; Schreiber 1998, S. 11 f.):

- In der Planungsphase ermittelt der/die selbstgesteuert Lernende den eigenen Lernbedarf, legt Ziele und Teilziele fest, wählt die zu bearbeitenden Lerninhalte und -aufgaben aus und identifiziert (menschliche und materielle) Ressourcen. Diese metakognitiven Aktivitäten werden begleitet von motivationalen und volitionalen Prozessen: Ausgehend von persönlichen Überzeugungen, z. B. Selbstwirksamkeitserwartungen, und zukunftsgerichteten Emotionen wie Angst, Hoffnung oder Vorfreude entwickelt er/sie eine Lernabsicht, für deren Umsetzung und Aufrechterhaltung entsprechende Vorkehrungen geplant werden (z. B. die Auswahl von Lernstrategien und die ablenkungsfreie Gestaltung der Lernumgebung).

- Die Durchführungsphase wird bestimmt von der Umsetzung der geplanten Aktivitäten in Lernhandlungen. Die zuvor ausgewählten kognitiven Lernstrategien werden nun angewendet, um neue Informationen aufzunehmen und zu verarbeiten und angemessen mit Ressourcen umzugehen. Zugleich sorgen metakognitive Aktivitäten für die fortlaufende Überwachung des Lernfortschritts und ggf. für die Anpassung der Lernhandlungen. Zur Anwendung kommen jetzt ebenfalls die volitionalen Komponenten, durch die die Lernabsicht gegenüber konkurrierenden Einflüssen z. B. emotionaler Art (Desinteresse, Langeweile) ab- 
geschirmt und der Lernprozess aufrechterhalten wird. Dies kann durch Aktivitäten auf der motivationalen Ebene unterstützt werden (z. B. Selbstbelohnung).

- Im Mittelpunkt der Bewertungsphase steht der dem metakognitiven Bereich zugehörige Vergleich der in der Durchführungsphase erzielten Handlungsresultate mit den in der Planungsphase festgelegten Lernzielen. Infolge dieser Bewertung, in die der/die selbstgesteuert Lernende ggf. auch weitere Personen einbezieht, können sich Emotionen wie Zufriedenheit, Stolz oder Enttäuschung einstellen, die Einfluss auf die Absichtsbildung im Rahmen künftiger Lernprozesse haben können (s.o.).

Am Ende solchermassen durchlaufener Lernprozesse stehen Lernergebnisse (oder «Lernprodukte») wie Wissens- und/oder Kompetenzzuwächse, Einsichten, Einstellungen etc.

Neben den oben geschilderten Selbststeuerungseinflüssen wirken sich weitere Variablen direkt oder indirekt auf den Lernprozess aus. So hängt etwa die konkrete Ausübung o.g. Aktivitäten in Qualität und Quantität von individuellen habituellen Lernermerkmalen ab. Diese Merkmale stellen relativ stabile, situationsunabhängige Grundeinstellungen des/der Lernenden in emotionaler, motivational-volitionaler, (meta-)kognitiver und sozialer Hinsicht dar. Beispielsweise kann Emotion nicht nur als momentaner Zustand (z. B. Prüfungsangst), sondern auch als zugrunde liegende überdauernde Verhaltensdisposition begriffen werden (Ängstlichkeit). Weitere lernbezogene Grundhaltungen sind unter Begriffen wie Lernmotive, Lernstile etc. bekannt. Die Lernermerkmale unterliegen - wie die entsprechenden Aktivitäten in den Lernprozessphasen - wechselseitigen Einflüssen.

Individuen entwickeln solche weit über Lernprozesse hinaus bedeutsamen Haltungen in der Interaktion mit ihrer Umwelt, also mit anderen Individuen, Institutionen, medial vermittelten Inhalten etc. Bezogen auf Lernen bilden Bildungssysteme, Lehr-/Lernkulturen, Bildungseinrichtungen, Lehrende, Mitlernende, Lernmedien und -inhalte etc. eine Lernumwelt, die die Einstellungen des/der Lernenden beeinflusst. Zugleich sind mit dem Begriff der Lernumwelt die konkreten fremdgesteuerten Einflüsse auf den Lernprozess angesprochen. Zu ihnen zählen z. B. situative, personelle und ggf. institutionelle Rahmenbedingungen wie inhaltliche, methodische und organisatorische Vorgaben/Vorschriften, das Verhalten von Lehrpersonen und Charakteristika des Gegenstandsbereichs sowie einzelner Aufgaben. Im verbindenden Element der Lernumwelt wird deutlich, dass die strikte Unterscheidung zwischen Selbst- und Fremdsteuerung vor allem analytischer Natur ist und die Begriffe darüber hinaus nicht objektivierbar sind (vgl. Faulstich 2002, S. 70; Reinmann-Rothmeier/Mandl 2001, S. 634).

Das in Abb. 1 dargestellte Modell greift die geschilderten lernbezogenen Einflussvariablen auf und veranschaulicht verschiedene Reflexionsebenen, die sich in den 
mit ihnen verbundenen Reflexionsgegenständen und -zielen unterscheiden und die im Folgenden kurz erläutert werden.

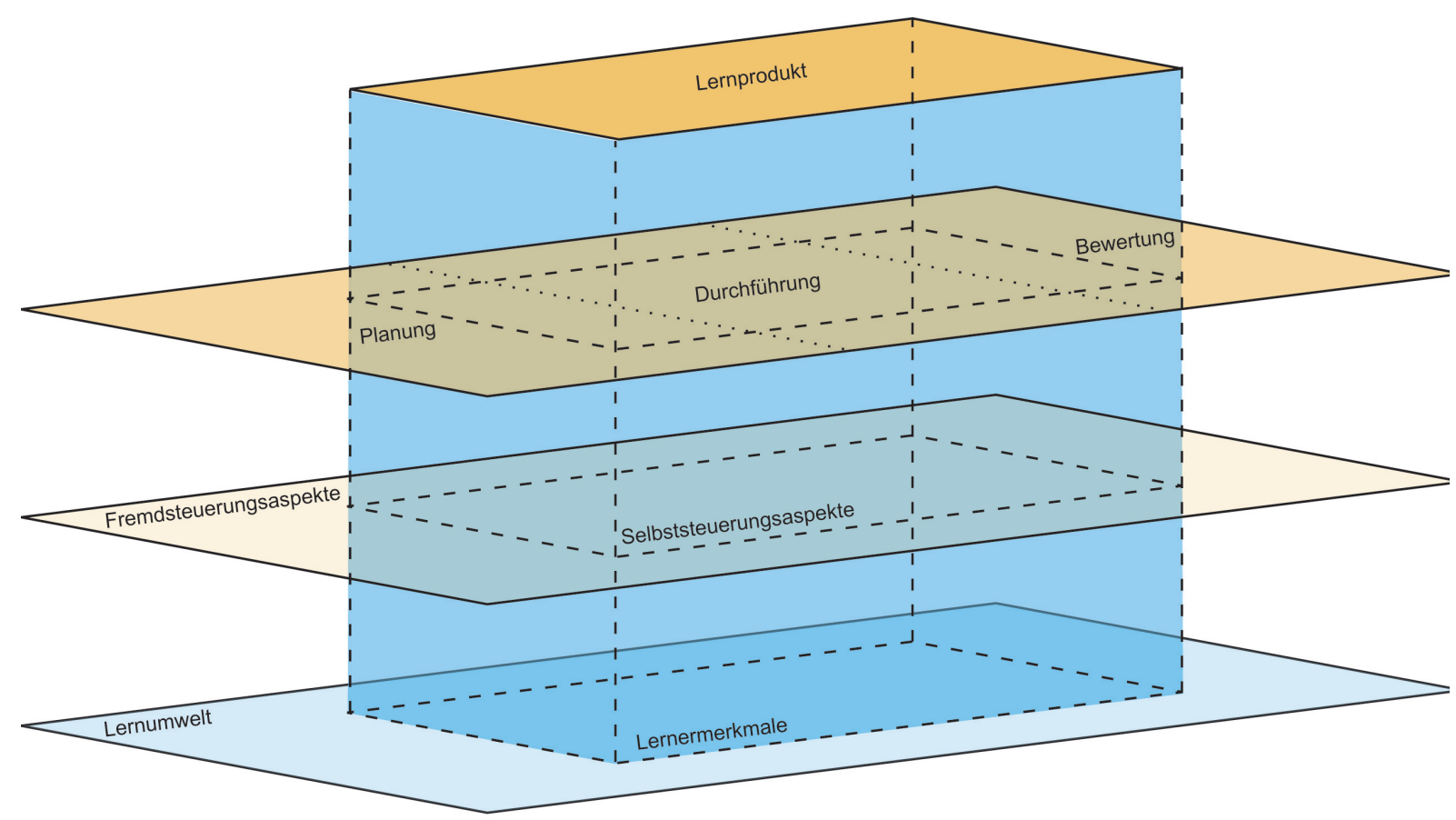

Abb. 1: Lernbezogene Einflussvariablen und Reflexionsebenen

\section{Ebenen lernbezogener Reflexion}

Auf der Ebene des Lernprozesses können Selbststeuerungseinflüsse, Fremdsteuerungseinflüsse und/oder die Prozessphasen als Bezugspunkte für Reflexion dienen. Stehen erstgenannte im Mittelpunkt, kann ggf. nach den einzelnen Aspekten (kognitive, metakognitive, emotionale, motivationale, volitionale) der Selbststeuerungsaktivitäten differenziert werden. Ähnlich verhält es sich mit den Fremdsteuerungseinflüssen; auch hier können ggf. die einzelnen Aspekte (situative, personelle, institutionelle Rahmenbedingungen) im Zentrum der Reflexion stehen. In beiden Fällen ergibt sich in Verbindung mit den Prozessphasen eine Vielzahl an Kombinationsmöglichkeiten und damit einhergehenden Reflexionsgegenständen. Ziel des unmittelbar im Lernprozess stattfindenden und auf Selbststeuerungsaktivitäten bezogenen Reflektierens ist die Optimierung dieser Aktivitäten und dadurch des Lernprozesses bzw. einzelner seiner Phasen.

Nicht zwingend an einen konkreten Lernprozess geknüpft ist Reflexion auf der Ebene der Lernermerkmale. Hier kann sich das Reflektieren des/der Lernenden auf einzelne Klassen von Grundeinstellungen (s. o.) oder auf deren Gesamtheit beziehen. Der/die Lernende kann sich z. B. grundlegender Lernziele und -motive oder 
emotionaler Haltungen bewusst werden, durch die sich evtl. förderliche und hinderliche internale Aspekte des Lernens erklären lassen. Reflexionsziel auf der Merkmalsebene kann beispielsweise die Erhöhung des individuellen Selbststeuerungspotenzials oder, allgemeiner ausgedrückt, die Entwicklung der (Lern-)Persönlichkeit sein.

Wird auch die Ebene der Lernumwelt in die Reflexion einbezogen, eröffnen sich weitere Zielperspektiven. Nur durch Mitbeachtung der allgemeinen Lernbedingungen als Gegenstand der Reflexion kann diese einem umfassenden Bildungsanspruch gerecht werden und z. B. die Urteilskraft Lernender fördern (vgl. Häcker/ Hilzensauer/Reinmann 2008, S. 1 f.).

Der durch selbst- und fremdgesteuerte Einflüsse direkt und durch individuelle und umweltbezogene Voraussetzungen indirekt beeinflusste Lernprozess mündet letztlich in ein Lernprodukt. Auch dieses kann zum Gegenstand von Reflexion werden, deren Ziel z. B. darin liegen kann, in Verbindung mit merkmalsbezogener Reflexion aktuelle individuelle Lern- und Entwicklungsbedarfe zu identifizieren.

\section{Wirkungen lernbezogener Reflexion}

Die Fähigkeit zum Reflektieren gilt als zentraler Erfolgsfaktor für selbstgesteuertes Lernen (s.o.), was angesichts der vielfach postulierten positiven Effekte lernbezogener Reflexion nicht verwundert. So wird ihr zugeschrieben, dass sie zur Optimierung instrumentellen Lernens und insgesamt zur Überwindung eines oft eingeengten Alltagsbewusstseins beiträgt: «Reflexives Lernen ermöglicht Distanz zu dem Bestehenden und fördert Phantasie für das Mögliche, es lässt die Welt als veränderbar erscheinen» (Siebert 1991, S. 25). Demgegenüber stehen die angenommenen negativen Folgen einer unzureichend ausgeprägten Reflexionsfähigkeit, die von Überforderung (als Folge zu seltenen Reflektierens, also fehlender Übung/Erfahrung, und mangelnder Unterstützung) über Spontaneitätseinbussen bis hin zur Handlungsunfähigkeit (aufgrund zu häufigen/intensiven Reflektierens) reichen (vgl. ebd., S. 21 ff.; Friedrich/Mandl 1992, S. 13). Empirische Untersuchungsergebnisse zu den Wirkungen lernbezogener Reflexion liefern Hinweise darauf, ob sich die an sie geknüpften Erwartungen tatsächlich erfüllen lassen.

Im Zuge der Lernstrategieforschung existieren bereits seit längerem Erkenntnisse zu Effekten des Reflektierens über Selbststeuerungsaktivitäten im Lernprozess. Festgestellt wurden umfangreiche positive Wirkungen vor allem hinsichtlich kognitiver Leistungen in der Durchführungsphase: Auf der Lernprozessebene Reflektierende «lösen Aufgaben schneller, passen sich besser an neue Verhältnisse an, erfassen Zusammenhänge, wiederholen Fehler nicht ein zweites Mal [und] sind eher zur Überprüfung ihrer Hypothesen bereit» (Siebert 1991, S. 25) als nicht in Reflexion geschulte Lernende oder solche, die Reflexion aus individuellen Gründen vermeiden (vgl. Dörner 1982, S. 145 f.; Friedrich/Mandl 1992, S. 13; Siebert 1991, S. 30 f.). Auch in jüngeren Studien wird von kognitiven Leistungssteigerun- 
gen und z.T. von Hinweisen auf einen verbesserten Lernstrategieeinsatz durch Reflexion berichtet (vgl. Gläser-Zikuda/Göhring 2007, S. 189 ff./S. 200 f.; Landmann/ Schmitz 2007, S. 142 ff.; Hübner/Nückles/Renkl 2007, S. 132 ff.). Für den Einsatz verschiedener, auch metakognitiver Strategien scheinen u. a. auch motivationalemotionale Faktoren ausschlaggebend zu sein: Studien belegen, dass eine hohe Selbstwirksamkeitserwartung generell positive Auswirkungen auf den Lernstrategieeinsatz hat (vgl. Friedrich/Mandl 1992, S. 25 f.; Schiefele/Pekrun 1996, S. 267). Der Einfluss von Selbstwirksamkeitserwartungen im und auf den konkreten Lernprozess lenkt den Blick zugleich auf die Ebene der Lernermerkmale. Reflexion auf dieser Ebene ist nur vereinzelt Gegenstand empirischer Untersuchungen, so z. B. bei Brouër (2007), die Studierende zum Reflektieren über ihre persönliche Entwicklung, die eigenen Stärken und Schwächen und ihre individuellen Ziele anregen wollte. Rückschlüsse auf Effekte derartig ausgerichteter Reflexion lässt die Studie leider nicht zu, da letztlich nur oberflächlich reflektiert wurde (vgl. ebd., S. 256). Die Annahme, Reflexion über persönliche Einstellungen könne zur Einstellungsänderung beitragen (vgl. Brahm/Seufert 2007, S. 10 f.), lässt sich damit zwar nicht belegen, bleibt aber vor dem Hintergrund der sich gegenseitig beeinflussenden Merkmale bzw. Merkmalsklassen (s.o.) plausibel.

Forschungsergebnisse zum Reflektieren Lernender auf der Ebene der Lernumwelt und/oder der der Fremdsteuerungsaspekte liegen nach Kenntnis der Autoren bisher nicht vor. Zwar werden die Rahmenbedingungen des Lernens und des Reflektierens auch in den erwähnten Untersuchungen thematisiert, jedoch nicht als Reflexionsgegenstand Lernender, sondern als Bestandteil der Schlussfolgerungen und Empfehlungen der jeweiligen Autor/innen.

\section{Rahmenbedingungen von Reflexion}

In verschiedenen Lernumwelten herrschen verschiedene (soziokulturelle, bildungspolitische etc.) Rahmenbedingungen, die sich in unterschiedlichen Fremdsteuerungseinflüssen niederschlagen. So ist es in einer eher auf rezeptives Lernen ausgerichteten Lernumwelt schwieriger als in einer für kreatives Lernen offenen, reflexive Elemente zu etablieren (vgl. Siebert 1991, S. 23); dies gilt sowohl auf institutioneller (z. B. mangelnde Unterstützungsangebote für Lernende) als auch auf individueller Ebene (z.B. ablehnende Einstellung der Beteiligten). Werden reflexive Anteile in organisierten Lehr-Lern-Kontexten zum Objekt formaler Bewertungen, besteht zudem die Gefahr, dass Lernende nicht authentisch, sondern einzig im Hinblick auf die zugrunde liegenden Bewertungskriterien «reflektieren». Doch auch das Nicht-Benoten kann unerwünschte Folgen wie z. B. nur oberflächliches Reflektieren haben (vgl. Brouër 2007, S. 256). Dies verdeutlicht die Notwendigkeit, die vielfältigen Rahmenbedingungen der Lernumwelt in sämtlichen Überlegungen zur Integration reflexiver Anteile im Lehr-Lern-Kontext zu berücksichtigen. 
Zur Förderung lernbezogener Reflexion scheint es grundsätzlich sinnvoll, den Lernenden umfassende Unterstützungsangebote zu unterbreiten und für Möglichkeiten zum Austausch der Lernenden z. B. mit Mitlernenden und Lehrenden zu sorgen (vgl. Brouër 2007, S. 258 ff.; Gläser-Zikuda/Göhring 2007, S. 201 ff.). Die genannten Punkte sind zentrale Elemente in Konzepten, die sich auf Portfolios als Medium zur Reflexionsunterstützung konzentrieren.

\section{4 (e)Portfolios als Reflexionsmedium}

Als gedanklicher Vorgang bedarf Reflexion prinzipiell keiner medialen Unterstützung. Erst das Abbilden der eigenen Überlegungen mittels eines Zeichensystems (Sprache, Schrift, Bild) eröffnet jedoch die Möglichkeit, die Gedanken anderen Personen mitzuteilen und sie sich ggf. zu einem späteren Zeitpunkt selbst erneut zu vergegenwärtigen. Im erziehungswissenschaftlichen Kontext haben Bemühungen, Lernende durch das selbsttätige Darstellen ihrer Lernprozesse und -leistungen zur Reflexion anzuregen, eine lange Tradition. Bereits reformpädagogische Ansätze weisen dem Anfertigen von Dokumentenmappen, Lerntagebüchern etc. diesbezüglich einen hohen Stellenwert zu (vgl. Häcker 2007a, S. 136 ff.). Aktuelle Konzepte greifen diese Überlegungen auf und stellen die Verwendung sog. Portfolios als persönliches Dokumentations- und Reflexionsmedium in den Mittelpunkt. Seit einigen Jahren kommen zunehmend auch sog. ePortfolios zum Einsatz, die von den Inhaber/innen nicht in Papierform, sondern mithilfe digitaler, webbasierter Medien erstellt werden und über spezifische Potenziale verfügen (s.u.).

In verschiedenen Ländern haben in den letzten Jahren Bestrebungen, Studierende durch eine lernerzentrierte, kompetenzorientierte Lehre zu lebenslangem Lernen zu befähigen, in Verbindung mit eLearning-Aktivitäten zu einer verstärkten ePortfolionutzung an Hochschulen geführt. Umfangreiche Erfahrungen werden z. B. in Grossbritannien seit der Verabschiedung einer nationalen eLearning-Strategie im Jahr 2005 gesammelt (vgl. HEA 2007; Beetham 2005), und auch in den Niederlanden werden ePortfolios durch die im Jahr 2004 gestartete «NL-Portfolio»-Initiative an zahlreichen Hochschulen eingesetzt (vgl. Aalderink/Veugelers 2005; SURF Foundation 2004). Ausserhalb Europas verfügen vor allem US-amerikanische Hochschulen über langjährige Erfahrungen mit (e)Portfolios, z. B. auch in dem für das im folgenden Kapitel beschriebene Projekt relevanten ingenieurwissenschaftlichen Bereich (vgl. Guan et al. 2006).

Trotz z.T. vorhandener nationaler Strategien/Initiativen können die didaktischen und technischen Rahmenbedingungen der (e)Portfolionutzung zwischen den Hochschulen stark variieren. Grundsätzlich wird jedoch immer die Förderung lern-/ entwicklungsbezogener Reflexion angestrebt. 


\section{Reflexion in der Portfolioarbeit}

In Bildungszusammenhängen verwendete Portfolios enthalten grundsätzlich eine von dem/der Lernenden zusammengestellte Auswahl von Dokumenten, die ihm/ ihr «die Darstellung der eigenen Entwicklung, des eigenen Könnens bzw. der eigenen Leistungen» (Häcker 2007a, S. 86) ermöglichen soll. Unabhängig von dieser Gemeinsamkeit können Portfolios sich in dem Zweck, den sie erfüllen sollen, deutlich unterscheiden und z. B. als alternatives Instrument zur Leistungsbewertung oder aber der Unterstützung der persönlichen Entwicklung Lernender dienen. Von Zweck und Ziel der Portfolioarbeit hängt auch ab, in welchem Ausmass Selbststeuerungsmöglichkeiten bestehen. Insbesondere in der Verwendung als Entwicklungsinstrument gilt das Reflektieren der Portfolioautor/innen über ihr Lernen und ihre Entwicklung als Herzstück des Portfolioprozesses, der dann letztlich darauf abzielt, sie zu selbstgesteuertem Lernen zu befähigen (vgl. ebd., S. 134 f.). Reflektiert wird bereits in der Phase der Sammlung möglicher Portfolioinhalte, da das Sammeln nicht beliebig, sondern ausgerichtet auf Zweck und Ziel der Portfolioarbeit erfolgt. Noch deutlicher im Vordergrund steht Reflexion in der der Sammelphase folgenden (oder, bei erfahreneren Portfolioautor/innen, mit ihr verbundenen) Phase der Auswahl von ins Portfolio aufzunehmenden Dokumenten. In der Regel hat der/die Portfolioautor/in hier die Aufnahme jedes Dokuments schriftlich zu begründen, indem er/sie kurz erläutert, was das Dokument über den Lernfortschritt, die Lernbedingungen oder das eigene Lernen insgesamt zeigt. Sowohl beim Sammeln als auch beim begründeten Auswählen benötigen insbesondere Portfolioanfänger/innen Unterstützung. Fester Bestandteil der als sozialer Prozess angelegten Portfolioarbeit ist die Möglichkeit des Austauschs zwischen Portfolioautor/innen untereinander sowie mit Lehrenden, anderen Expert/innen, Peers und weiteren Personen. Dieser Austausch ermöglicht es allen Beteiligten, andere Perspektiven kennenzulernen, und trägt so zur Reflexion bei (vgl. ebd., S. 145 ff.). Gerade der kooperative und kommunikative Charakter der Portfolioarbeit kann Wirkungen auf unterschiedlichen Ebenen hervorrufen. An Beispielen projektorientierten Schulunterrichts zeigt Häcker (2007a), dass nicht nur Schüler/innen in unterschiedlicher Weise von der Arbeit mit Portfolios profitieren können und sie u. a. das Nachdenken über ihr Lernen als sinnvoll empfinden, sondern dass zahlreiche Impulse auch auf Seiten der Lehrer/innen und der Unterrichtsebene festzustellen sind (vgl. S. 228 ff./S. 296 ff.).

\section{Potenziale von ePortfolios}

Je nach Zweck eines ePortfolios können Content-Management-Systeme, SocialSoftware-Anwendungen oder auch spezielle ePortfoliotools zum Einsatz kommen. Die Möglichkeiten der digitalen Medien können sich auf Inhalt und Struktur des Portfolios und auf die mit ihm verbundenen Prozesse auswirken: ePortfolios ermöglichen die Aufnahme multimedialer Inhalte, können die Darstellung von Ver- 
bindungen/Beziehungen zwischen Inhalten sowie den Zugriff auf diese unterstützen (z. B. durch Links, Schlagwörter und Suchfunktionen) und lassen sich schneller und extensiver verbreiten als herkömmliche Portfolios. Insbesondere die kommunikative und kooperative Komponente der Portfolioarbeit kann durch eine detaillierte Zugriffs-/Rechtverwaltung und ein integriertes Feedbacksystem unterstützt werden (vgl. Hornung-Prähauser et al. 2007, S. 27 ff.; Schaffert et al. 2007, S. 77 ff.). Inwieweit die genannten Potenziale jedoch auch ausgeschöpft werden und ob sie sich, wie z.T. behauptet, positiv auf Reflexion, Motivation oder Medienkompetenz der Portfolioautor/innen auswirken, kann bislang nicht beurteilt werden, da in Studien zu ePortfolios die Perspektive der Lernenden zu selten berücksichtigt wird (vgl. Brahm/Seufert 2007, S. 16).

\section{Reflexionsunterstützung in (e)Portfolios}

Trotz der zentralen Funktion von Reflexion in der Portfolioarbeit finden sich in entsprechenden Anleitungen bestenfalls sehr allgemein gehaltene Empfehlungen zum Reflektieren; selbst in explizit als ePortfoliosoftware ausgewiesenen Anwendungen fehlen derartige Beschreibungen oder Anregungen (vgl. Hilzensauer 2008, S. 12 ff.). Zwar können detaillierte Anweisungen nicht erwartet werden, da in die Gestaltung der Portfolioarbeit immer die jeweiligen Rahmenbedingungen der Lernumwelt einfliessen müssen (s.o.), dennoch könnten zumindest grundlegende methodische Hinweise z.B. auf mögliche Reflexionsziele und -ebenen/-gegenstände hilfreich sein. Anregen lässt sich Reflexion beispielsweise durch Leitfragen, die dem/der Autor/in an die Hand gegeben werden und auf die er/sie erst gedanklich, dann schriftlich Antworten formulieren kann. Derartige Leitfragen sind in der (e)Portfolioarbeit keine Seltenheit. Sie zielen zumeist auf den Lernprozess und/ oder das Lernprodukt ab, während Lernermerkmale und die Lernumwelt i.d.R. nicht thematisiert werden. Aus Erfahrungsberichten und empirischen Studien geht hervor, dass sich Leitfragen grundsätzlich dazu eignen, Reflexion auf der Lernprozess- und der Lernproduktebene anzuregen (vgl. Brouër 2007, S. 251 ff.; Pölzleitner 2008, S. 96 ff.; Hübner/Nückles/Renkl 2007, S. 126; Volkwein 2008, S. 152 f.; Schelbert 2008, S. 134 f.). Interindividuelle Unterschiede haben jedoch starken Einfluss auf die Wirkung von Leitfragen; so scheinen sie z. B. als Hilfestellung für im Reflektieren eher ungeübte Lernende geeignet zu sein, während geübtere sie offenbar eher als störend oder hemmend empfinden (vgl. Hübner/Nückles/Renkl 2007, S. $130 \mathrm{ff}$.). Auch dies weist auf die Notwendigkeit, Rahmenbedingungen zu berücksichtigen, hin (s. o.).

\section{Reflexionsförderung im ePortfoliosystem der TU Hamburg-Harburg}

Seit Mitte 2008 läuft an der Technischen Universität Hamburg-Harburg (TUHH) das vom eLearning Consortium Hamburg bis Ende 2010 geförderte Projekt «studlPort 2.0», an dessen Durchführung verschiedene TUHH-Institute und -Einrichtungen 
(Rechenzentrum, Bibliothek) mitwirken. Im Folgenden werden die didaktischen Projektziele, die Grundlagen der ePortfolioarbeit und das ePortfoliosystem an der TUHH sowie die Massnahmen zur Unterstützung von Reflexionsprozessen kurz erläutert.

\section{Didaktische Projektziele}

Die didaktischen Projektziele dienen sämtlich der Unterstützung individueller Lernprozesse der TUHH-Studierenden (ca. 5.000 in über 40 grösstenteils ingenieurwissenschaftlichen Studiengängen). Im Einzelnen sollen Eigenverantwortung und Selbststeuerung gefördert und insbesondere Selbstreflexion sowohl im Hinblick auf Lernprozesse als auch auf die persönliche Lernbiografie angeregt werden, darüber hinaus wird die Stärkung des Lernens im Austausch angestrebt. Als hinsichtlich dieser Ziele strukturbildende Massnahme wird an der TUHH ein hochschulweites ePortfoliosystem eingeführt. Vor dem Hintergrund der Rahmenbedingungen des Projekts und der Recherchen zur ePortfolioarbeit an Hochschulen im In- und Ausland, insbesondere in der Ingenieursausbildung, wurden die Grundsätze der ePorfolioarbeit an der TUHH festgelegt.

\section{ePortfolioarbeit an der TUHH}

In didaktischer Hinsicht wurde zunächst entschieden, dass das ePortfoliosystem im Rahmen des Projekts a) als freiwillig nutzbares Angebot konzipiert und b) nicht zur formalen Leistungsbewertung eingesetzt werden soll. Als Basis für die Detailplanung des ePortfoliosystems dienten Beschreibungen grundlegender studierendenzentrierter Prozesse und Szenarien der ePortfolioarbeit an der TUHH (s. Abb. 2):

1 Die Studierenden reflektieren die Erfahrungen und Kompetenzen, die sie in verschiedenen Lebensbereichen sammeln bzw. erwerben, um sich persönlicher Stärken, Schwächen, Vorlieben, Abneigungen und auch (Studien-/Berufs-)Erwartungen bewusst(er) zu werden.

2 In ihrem ePortfolio beschreiben die Studierenden ihre Kompetenzen bzw. belegen sie mithilfe selbst ausgewählter - und möglichst auch selbst angefertigter - Dateien. Auch alle weiteren Erkenntnisse aus der Reflexionsphase halten sie dort fest. Anregungen zur Überarbeitung der ePortfolioinhalte können optionale Reflexions- und Feedbackschleifen, in die ggf. verschiedene Personen(gruppen) einbezogen werden, liefern.

Den Zweck der ePortfolioarbeit legen die Studierenden individuell selbst fest. Als Anregung können drei Szenarien dienen, die die Projektgruppe formuliert hat und auf die Unterstützungs- und Marketingmassnahmen ausgerichtet werden: 
1 Im Szenario Orientierung nutzen die Studierenden ihr ePortfolio, um zu Beginn ihres Studiums die für sie passenden Studienschwerpunkte zu identifizieren. Das Szenario Bewerbung gewinnt im fortgeschrittenen Studium an Bedeutung, hier steht die Präsentation der eigenen Persönlichkeit bei der Bewerbung um einen Arbeits- oder Praktikumsplatz im Vordergrund.

2 Im Szenario Entwicklung verwenden Studierende ihr ePortfolio unabhängig von an bestimmte Zeitpunkte geknüpften Zwecken, sondern führen die beschriebenen Prozesse dauerhaft mit dem Ziel der persönlichen Weiterentwicklung fort.

Zu den Aufgaben der Projektgruppe zählt insbesondere die Unterstützung von Reflexions- und Feedbackprozessen; angestrebt wird darüber hinaus ein dauerhafter Erfahrungsaustausch zwischen allen an der ePortfolioarbeit Beteiligten.

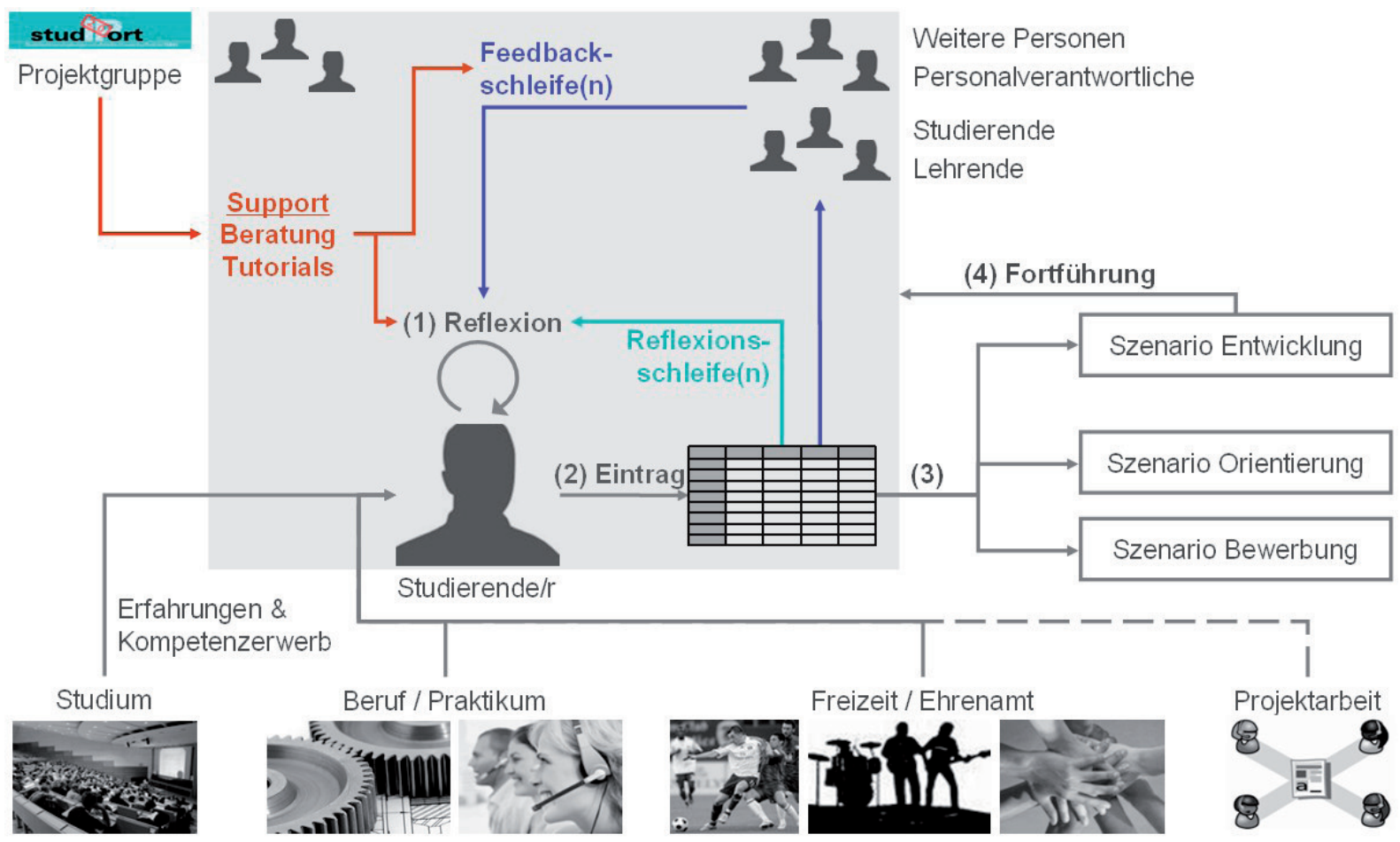

Abb. 2: Grundlegende Prozesse und Szenarien der ePortfolioarbeit an der TUHH

\section{Das TUHH-ePortfoliosystem}

Wie eine Begutachtung verschiedener frei zugänglicher, z. T. explizit als ePortfoliosoftware ausgewiesener Systeme ergab, erfüllte keines davon die aus den beschriebenen Prozessen und Szenarien resultierenden technischen Anforderungen 
an das TUHH-ePortfoliosystem. Es wurde folglich entschieden, ein eigenes ePortfoliosystem zu entwickeln und dieses in die an der TUHH verwendete Lernplattform Stud.IP zu integrieren.

Das Kernelement im TUHH-ePortfolio ist die sog. Kompetenzmatrix, die das strukturierte Sammeln und Darstellen von ePortfolioinhalten unterstützt (s. Abb. 3). In der Matrix kann jede Datei, die der/die Studierende in das ePortfolio aufnimmt, Kompetenz- und Lebens-/Erfahrungsbereichen zugeordnet und mit einer Beschreibung sowie weiteren Anmerkungen versehen werden. Während die Datei später mitsamt ihrer Beschreibung anderen Personen zugänglich gemacht werden kann, bleiben die weiteren Anmerkungen ausschliesslich dem/der Studierenden sichtbar. Beschreibungen und Anmerkungen können nicht nur einzelnen Dateien, sondern auch Kompetenz- und Lebensbereichen hinzugefügt werden. Die Kompetenzbereiche wurden im Rahmen einer breit angelegten Dokumentenanalyse und anschliessender Befragungen der TUHH-Studierenden und -Lehrenden sowie von Vertreter/innen potenzieller Arbeitgeber für TUHH-Absolvent/innen ermittelt.

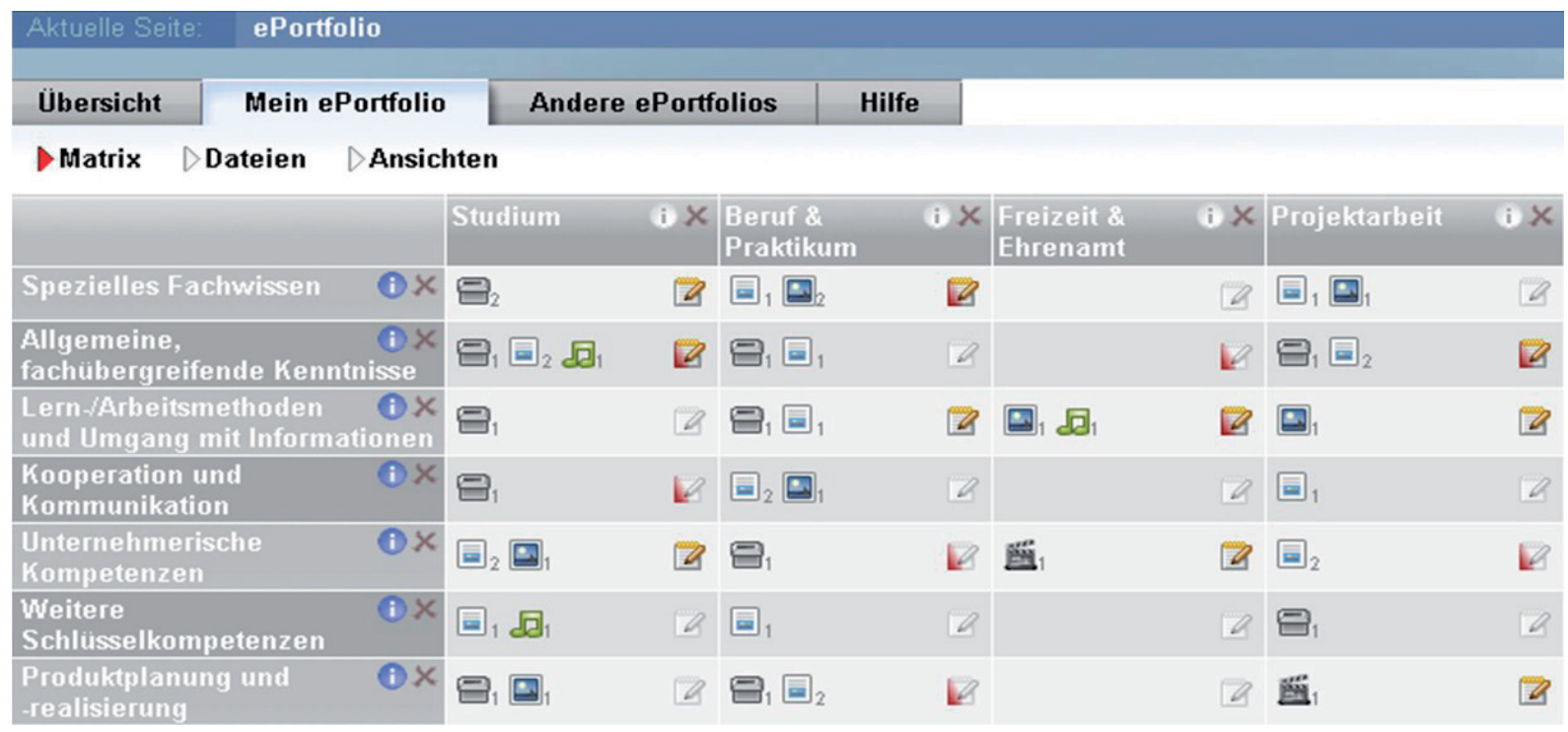

Abb. 3: Die (beispielhaft gefüllte) Kompetenzmatrix im ePortfolio-Prototyp der TUHH

Weitere zentrale Komponenten im TUHH-ePortfolio sind das Ansichten- und das Feedbacksystem. Erstgenanntes ermöglicht das Zusammenstellen von ePortfolioinhalten zu sog. Ansichten, die bestimmten Personen für festgelegte Zeiträume zugänglich gemacht werden können, Letzteres dient dem Formulieren ansichtenspezifischer Fragen für die zugriffsberechtigten Personen. Der/die Studierende hat somit zu jeder Zeit die volle Kontrolle darüber, wer wann unter welcher Fragestellung auf welche ePortfolioinhalte zugreifen darf. Zu allen ePortfoliofunktionen 
werden über eine kontextsensitive Hilfefunktion ausführliche Informationen und Hinweise angeboten.

\section{Reflexionsunterstützung im TUHH-ePortfolio}

Wie erwähnt liegt eine der Hauptaufgaben der Projektgruppe in der Reflexionsunterstützung. Da sich Reflexion auf verschiedene Ebenen beziehen kann (s. o.), gilt es in einem ersten Schritt, die im konkreten Zusammenhang bedeutsamen Ebenen zu identifizieren. Im hier beschriebenen Projekt bedingt die Verwendung von ePortfolios Reflexion auf der Ebene des Lernprodukts (durch das Sammeln und Auswählen entsprechender Inhalte). Explizit in den Projektzielen angesprochen werden Selbststeuerungsanteile auf der Ebene des Lernprozesses, jedoch weder begrenzt auf einzelne Phasen oder Aspekte noch zwingend auf ein Lernprodukt bezogen. Für die ebenfalls in den Zielen genannte Auseinandersetzung mit der eigenen Lernbiografie scheint Reflexion auf der Ebene der Lernermerkmale wie auch auf der der Lernumwelt relevant. Auf den genannten Ebenen soll Reflexion mithilfe folgender Leitfragen unterstützt werden:

- Ebene Lernprodukt:

- (Sammlung) Warum sollte ich diese Datei in mein ePortfolio aufnehmen? Was würde ihm fehlen, wenn ich sie nicht aufnehmen würde?

- (Auswahl) Was sagt diese Datei über meine Fähigkeiten und Kenntnisse aus? Zu welchem Kompetenz- und Erfahrungsbereich meiner Matrix passt sie am besten - und warum?

- Ebene Lernprozess:

- (Planung) Mit welchen Zielen habe ich die Datei angefertigt? Was wollte ich nachher können, wissen oder haben, was ich vorher nicht konnte, wusste oder hatte?

- (Durchführung) Wie bin ich beim Lernen/Arbeiten vorgegangen?

- (Durchführung) Welche Erwartungen, Hoffnungen oder Befürchtungen hatte ich beim Lernen/Arbeiten? War ich mir z. B. zu jedem Zeitpunkt sicher, die Ziele erreichen zu können?

- (Bewertung) Was lief gut und wo hatte ich Schwierigkeiten? Was hätte ich anders machen können, damit es noch besser funktioniert?

- (Bewertung) Welche meiner Ziele habe ich erreicht, welche nicht? Welche «unbeabsichtigten» Wirkungen wie neue Einstellungen/Meinungen etc. haben sich ggf. ergeben?

- (Bewertung) Wie zufrieden bin ich rückblickend mit meinem Lern-/Arbeitsprozess? 
- Ebene Lernermerkmale:

- Wie möchte ich mich (hinsichtlich dieses Kompetenz- und/oder Erfahrungsbereichs) entwickeln? Was möchte ich zukünftig können, wissen oder haben?

- Was kann ich tun (bzw. tue ich bereits), um diese Ziele zu erreichen?

- Welche meiner Charaktereigenschaften helfen mir dabei, meine Ziele zu erreichen? Und welche sind eher hinderlich? Welche Erfahrungen habe ich diesbezüglich in der Vergangenheit gemacht?

- Ebene Lernumwelt:

- Welche äusseren Bedingungen (im Studium/Praktikum/Job/Privatleben) empfinde ich im Hinblick auf meine persönlichen Ziele als hilfreich? Und welche behindern mich?

- Wie kann ich die äusseren Bedingungen beeinflussen, damit sie mir noch mehr helfen bzw. mich weniger behindern?

Die Leitfragen sind Bestandteil des erwähnten Hilfesystems, so dass die Studierenden sie sich bei Bedarf jederzeit ansehen und darüber entscheiden können, welche der Fragen sie als Anregung zur Reflexion nutzen möchten.

\section{Ausblick}

Der Prototyp des TUHH-ePortfolios wird bis Januar 2010 von einer Gruppe interessierter Studierender der TUHH getestet. Ihre Erfahrungen und Anregungen fliessen in die Weiterentwicklung des Systems ein, das ab dem Sommersemester 2010 allen TUHH-Studierenden zur Verfügung stehen soll. Begleitet wird die breite Einführung des ePortfoliosystems von umfangreichen Marketing-, Informations- und Evaluationsmassnahmen, die zu einer möglichst umfassenden Akzeptanz des Systems beitragen sollen. Im Austausch mit allen Beteiligten wird die Projektgruppe insbesondere ermitteln, inwieweit die ePortfolioarbeit als reflexionsfördernd wahrgenommen wird, wie die Beteiligten den Stellenwert der Reflexionsanteile beurteilen und wie die unterstützenden Angebote empfunden werden. Eine darauf abgestimmte Gestaltung des ePortfoliosystems kann einen wesentlichen Beitrag zur Vorbereitung der Studierenden auf selbstgesteuertes lebenslanges Lernen leisten.

\section{Fazit}

Die Fähigkeit zur Selbststeuerung des Lernens gilt als Grundlage erfolgreichen lebenslangen Lernens. Selbststeuerung setzt ihrerseits Reflexionsfähigkeit voraus: Lernende müssen sich ihres Lernens bewusst werden, um es angemessen steuern zu können. Wie gezeigt wurde, kann sich die Reflexion des eigenen Lernens auf verschiedene Ebenen (und dort ggf. auf bestimmte Aspekte) beziehen und positi- 
ve Wirkungen auf Seiten der Lernenden hervorrufen. Das dargestellte Mehrebenenmodell erleichtert das Identifizieren möglicher Reflexionsziele und -gegenstände.

(e)Portfolios haben das Potenzial, Reflexion auf sämtlichen Ebenen anzuregen. Stehen Reflexionsziele und -gegenstände fest, können Lernende z. B. mithilfe von Leitfragen beim Reflektieren unterstützt werden. Dieser Weg wurde im beschriebenen Projekt an der Technischen Universität Hamburg-Harburg gewählt, in welchem gemäss den didaktischen Projektzielen zunächst ein umfassendes, alle Reflexionsebenen betreffendes Reflektieren angestrebt wird. Die Wirkungen der Projektmassnahmen können zum jetzigen Zeitpunkt zwar noch nicht benannt werden, in jedem Fall wird das Projekt jedoch dazu beitragen, zwei bestehende Defizite abzubauen: zum einen den Mangel an Studien zu ePortfolios, die die Perspektive der Lernenden berücksichtigen, und zum anderen das häufige Fehlen reflexionsunterstützender Elemente in der ePortfolioarbeit.

\section{Literatur}

Aalderink, Wijnand; Veugelers, Marij (2005). E-portfolios in the Netherlands: Stimulus for educational Change and lifelong learning: http://www.surfspace.nl/en/ Themas/portfolio/Start/Documents/helsinki_eden_-_wijnand_aalderinkmarij_ veugelers.pdf (17.11.2009).

Arnold, Rolf (1993). Natur als Vorbild. Reihe Wissenschaft in gesellschaftlicher Verantwortung, Band 26. Frankfurt/Main: VAS.

Arnold, Rolf; Gómez Tutor, Claudia (2007). Grundlinien einer Ermöglichungsdidaktik. Bildung ermöglichen - Vielfalt gestalten. Augsburg: ZIEL Verlag.

Bastian, Johannes (2003). «Selbstgesteuertes Lernen. Editorial.» Pädagogik 55.5: 3.

Beetham, Helen (2005). e-portfolios in post-16 learning in the UK: developments, issues and opportunities: http://www.jisc.ac.uk/media/documents/themes/ elearning/eportfolioped.pdf (17.11.2009).

BLK Bund-Länder-Kommission für Bildungsplanung und Forschungsförderung (2004). Strategie für Lebenslanges Lernen in der Bundesrepublik Deutschland. Materialien zur Bildungsplanung und zur Forschungsförderung, Heft 115. Bonn: BLK.

Boekaerts, Monique (1999). «Self-regulated learning: where we are today.» International Journal of Educational Research 31: 445-457.

Brouër, Birgit (2007). «Portfolios zur Unterstützung der Selbstreflexion - Eine Untersuchung zur Arbeit mit Portfolios in der Hochschullehre.» Lernprozesse dokumentieren, reflektieren und beurteilen. Lerntagebuch und Portfolio in Bildungsforschung und Bildungspraxis. Hrsg. v. Michaela Gläser-Zikuda u. Tina Hascher. Bad Heilbrunn: Verlag Julius Klinkhardt, 235-265. 
Dörner, Dietrich (1982). «Lernen des Wissens- und Kompetenzerwerbs.» Lehr-LernForschung. Ein Überblick in Einzeldarstellungen. Hrsg. v. Bernhard Treiber u. Franz E. Weinert. München, Wien, Baltimore: Urban \& Schwarzenberg, 134148.

Dohmen, Günther (1999). Weiterbildungsinstitutionen, Medien, Lernumwelten: Rahmenbedingungen und Entwicklungshilfen für das selbstgesteuerte Lernen. Bonn: Bundesministerium für Bildung und Forschung, Referat Öffentlichkeitsarbeit.

Faulstich, Peter (2002). "Vom selbstorganisierten zum selbstbestimmten Lernen.» Praxishandbuch selbstbestimmtes Lernen. Hrsg. v. Peter Faulstich. Weinheim, München: Juventa, 61-98.

Faulstich, Peter; Zeuner, Christine (1999). Erwachsenenbildung. Eine handlungsorientierte Einführung. Weinheim, München: Juventa.

Friedrich, Helmut F.; Mandl, Heinz (1992). «Lern- und Denkstrategien - ein Problemaufriss.» Lern- und Denkstrategien. Analyse und Intervention. Hrsg. v. Heinz Mandl u. Helmut F. Friedrich. Göttingen: Hogrefe, 3-54.

Friedrich, Helmut F.; Mandl, Heinz (1997). «Analyse und Förderung selbstgesteuerten Lernens.» Psychologie der Erwachsenenbildung. Hrsg. v. Franz E. Weinert u. Heinz Mandl. Göttingen: Hogrefe, 237-295.

Gläser-Zikuda, Michaela; Göhring, Anja (2007). «Analyse und Förderung selbstregulierten Lernens auf der Grundlage des Portfolio-Ansatzes - ein Forschungsprogramm in der Sekundarstufe I.» Lerntagebuch und Portfolio auf dem Prüfstand. Empirische Pädagogik, Themenheft 21 (2). Hrsg. v. Michaela Gläser-Zikuda. Landau: Verlag Empirische Pädagogik, 174-208.

Guan, Zhiwei; Lappenbusch, Steve; Turns, Jennifer; Yellin, Jessica (2006). »Portfolios in Engineering Education: What do they promise and how can they be used?" ASEE Conference Proceedings 2006: http://soa.asee.org/paper/conference/paper-view.cfm?id=2060 (17.11.2009).

Häcker, Thomas (2007a). Portfolio: ein Entwicklungsinstrument für selbstbestimmtes Lernen. Eine explorative Studie zur Arbeit mit Portfolios in der Sekundarstufe I. Baltmannsweiler: Schneider Verlag Hohengehren GmbH.

Häcker, Thomas (2007b). «Portfolio - ein Medium im Spannungsfeld zwischen Optimierung und Humanisierung des Lernens.» Lernprozesse dokumentieren, reflektieren und beurteilen. Lerntagebuch und Portfolio in Bildungsforschung und Bildungspraxis. Hrsg. v. Michaela Gläser-Zikuda u. Tina Hascher. Bad Heilbrunn: Verlag Julius Klinkhardt, 63-85.

Häcker, Thomas; Hilzensauer, Wolf; Reinmann, Gabi (2008). «Editorial zum Schwerpunktthema Reflexives Lernen.» bildungsforschung 5.2: http://www.bildungsforschung.org/Archiv/2008-02/editorial/ (17.11.2009).

HEA The Higher Education Academy (2007). A survey of e-pdp and e-portfolio practice in UK Higher Education: http://www.heacademy.ac.uk/assets/York/ 
documents/ourwork/tla/personal_development_plan/survey_of_epdp_and_ eportfolio_practice_in_uk_higher_education.pdf (17.11.2009).

Hilzensauer, Wolf (2008). «Theoretische Zugänge und Methoden zur Reflexion des Lernens. Ein Diskussionsbeitrag.» bildungsforschung 5.2: http://www.bildungsforschung.org/Archiv/2008-02/lernvermoegen/ (17.11.2009).

Hornung-Prähauser, Veronika; Geser, Guntram; Hilzensauer, Wolf; Schaffert, Sandra (2007). Didaktische, organisatorische und technologische Grundlagen von E-Portfolios und Analyse internationaler Beispiele und Erfahrungen mit E-Portfolio-Implementierungen an Hochschulen. Salzburg: Salzburg Research Forschungsgesellschaft.

Hübner, Sandra; Nückles, Matthias; Renkl, Alexander (2007). «Lerntagebücher als Medium des selbstgesteuerten Lernens - Wie viel instruktionale Unterstützung ist sinnvoll?» Lerntagebuch und Portfolio auf dem Prüfstand. Empirische Pädagogik, Themenheft 21 (2). Hrsg. v. Michaela Gläser-Zikuda. Landau: Verlag Empirische Pädagogik, 119-137.

Landmann, Meike; Schmitz, Bernhard (2007). «Nutzen und Grenzen standardisierter Selbstregulationstagebücher.» Lerntagebuch und Portfolio auf dem Prüfstand. Empirische Pädagogik, Themenheft 21 (2). Hrsg. v. Michaela Gläser-Zikuda. Landau: Verlag Empirische Pädagogik, 138-156.

Pölzleitner, Elisabeth (2008). «Reflektieren kann man lernen. Formblätter als Hilfe zur Selbsteinschätzung.» Das Handbuch Portfolioarbeit. Konzepte, Anregungen, Erfahrungen aus Schule und Lehrerbildung. Hrsg. v. Ilse Brunner, Thomas Häcker u. Felix Winter. Seelze-Velber: Kallmeyer, 96-111.

Reinmann-Rothmeier, Gabi; Mandl, Heinz (2001). «Unterrichten und Lernumgebungen gestalten.»Pädagogische Psychologie. 4., vollständig überarbeitete Auflage. Hrsg. v. Andreas Krapp u. Bernd Weidenmann. Weinheim: Beltz PVU, 601-646.

Schaffert, Sandra; Hornung-Prähauser, Veronika; Hilzensauer, Wolf; Wieden-Bischof, Diana (2007). «E-Portfolio-Einsatz an Hochschulen: Möglichkeiten und Herausforderungen.» 〈Ne(x)t Generation Learning): E-Assessment und E-Portfolio: Halten sie, was sie versprechen? SCIL-Arbeitsbericht 13. Hrsg. v. Taiga Brahm u. Sabine Seufert. St. Gallen: SCIL, 74-89. www.salzburgresearch.at/research/ gfx/brahm-seufert-next-generation-learning.pdf (24.3.2010).

Schelbert, Beat (2008). «Das Talentportfolio - eine Schatztruhe der Stärken.» Das Handbuch Portfolioarbeit. Konzepte, Anregungen, Erfahrungen aus Schule und Lehrerbildung. Hrsg. v. Ilse Brunner, Thomas Häcker u. Felix Winter. Seelze-Velber: Kallmeyer, 127-135.

Schiefele, Ulrich; Pekrun, Reinhard (1996). «Psychologische Modelle des fremdgesteuerten und selbstgesteuerten Lernens.» Psychologie des Lernens und der Instruktion. Hrsg. v. Franz E. Weinert. Göttingen: Hogrefe, 249-278. 
Schreiber, Beate (1998). Selbstreguliertes Lernen: Entwicklung und Evaluation von Trainingsansätzen für Berufstätige. Münster: Waxmann.

Seufert, Sabine; Brahm, Taiga (2007). «E-Assessment und E-Portfolio zur Kompetenzentwicklung: neue Potenziale für $\mathrm{Ne}(\mathrm{x}) \mathrm{t}$ Generation Learning.» « $\mathrm{Ne}(\mathrm{x}) \mathrm{t}$ Generation Learning): E-Assessment und E-Portfolio: Halten sie, was sie versprechen? SCILArbeitsbericht 13. Hrsg. v. Taiga Brahm und Sabine Seufert. St. Gallen: SCIL, 2-26. www.scil.ch/fileadmin/Container/Leistungen/Veroeffentlichungen/200703-brahm-seufert-next-generation-learning.pdf (24.3.2010).

Siebert, Horst (1991). "Aspekte einer reflexiven Didaktik.» Zehn Jahre Erwachsenenbildungswissenschaft. Reihe Theorie und Praxis der Erwachsenenbildung. Hrsg. v. Wilhelm Mader u. a. Bad Heilbrunn/Obb.: Klinkhardt, 19-32.

Siebert, Horst (2006). Didaktisches Handeln in der Erwachsenenbildung. Didaktik aus konstruktivistischer Sicht. 5., überarbeitete Auflage. Augsburg: ZIEL.

SURF Foundation (2004). Electronic Portfolio in the Netherlands. Some Articles: http://www.surfspace.nl/en/Themas/portfolio/Start/Documents/Electronic_ Portfolio_in_the_Netherlands.pdf (17.11.2009).

Volkwein, Karin (2008). "lch seh' den Text jetzt mit anderen Augen. Das Portfolio als Medium reflexiven Lernens.» Das Handbuch Portfolioarbeit. Konzepte, Anregungen, Erfahrungen aus Schule und Lehrerbildung. Hrsg. v. Ilse Brunner, Thomas Häcker u. Felix Winter. Seelze-Velber: Kallmeyer, 151-155.

Wild, Elke; Hofer, Manfred; Pekrun, Reinhard (2001). «Psychologie des Lerners.» Pädagogische Psychologie. 4., vollständig überarbeitete Auflage. Hrsg. v. Andreas Krapp u. Bernd Weidenmann. Weinheim: Beltz PVU, 207-269.

Wyss, Corinne (2008). «Zur Reflexionsfähigkeit und -praxis der Lehrperson.» bildungsforschung 5.2: http://www.bildungsforschung.org/Archiv/2008-02/lehrperson/ (17.11.2009). 